\title{
Conductance spectra of metallic carbon nanotube bundles from first principles
}

\author{
Wei Ren, ${ }^{1}$ C. T. Chan, ${ }^{1}$ T. H. Cho, ${ }^{2}$ T. C. Leung, ${ }^{2}$ Jian Wang, ${ }^{3}$ Hong Guo, ${ }^{4}$ and Ping Sheng ${ }^{1}$ \\ ${ }^{1}$ Department of Physics, Hong Kong University of Science and Technology, Clear Water Bay, Kowloon, Hong Kong, China \\ ${ }^{2}$ Department of Physics, National Chung Cheng University, Chia-Yi, Taiwan 62101, Republic of China \\ ${ }^{3}$ Center of Theoretical and Computational Physics and Department of Physics, The University of Hong Kong, \\ Pokfulam Road, Hong Kong, China \\ ${ }^{4}$ Department of Physics, McGill University, Montreal, Quebec, Canada H3A2T5
}

(Received 29 September 2008; published 4 November 2008)

\begin{abstract}
We report a first-principles analysis of electronic transport characteristics for $(n, n)$ carbon nanotube bundles. When $n$ is not a multiple of 3, intertube coupling causes universal conductance suppression near Fermi level regardless of the rotational arrangement of individual tubes. However, when $n$ is a multiple of 3 , the bundles exhibit a diversified conductance dependence on the orientation details of the constituent tubes. The total energy of the bundle is also sensitive to the orientation arrangement only when $n$ is a multiple of 3 . All the transport properties and band structures can be well understood from the symmetry consideration of whether the rotational symmetry of the individual tubes is commensurate with that of the bundle. This finding may shed some light on understanding and applications of nanotube bundle interconnect technology.
\end{abstract}

DOI: 10.1103/PhysRevB.78.195404

PACS number(s): 73.63.-b, 61.46.Fg, 71.20.-b

Single walled carbon nantubes (SWNTs) can form a bundle with a triangular cross-sectional lattice in a selforganized manner, a good example of hierarchical solids. The number of SWNTs in a bundle ranges from a few to hundreds. It has been known that close-packed bundles containing parallel individual nanotubes possess superb mechanic, ${ }^{1}$ thermal, ${ }^{2}$ and electronic ${ }^{3-5}$ properties. Since nanotube bundles are ubiquitous in nanotube synthesis reaction, they have attracted great attention both experimentally and theoretically. Very recently it was determined that the minimal bundling number is between 3 and 8 in vertically aligned SWNT films. ${ }^{6}$ For these bundles containing small number of tubes, the one-dimensional (1D) properties of SWNT can still be attainable. This is in contrast to bundles with large number of tubes where bulk properties might dominate.

SWNT bundles as nanowires have exciting prospect for nanoelectronics. In particular, metallic nanotube bundles have been proposed both theoretically and experimentally ${ }^{7}$ to outperform conventional copper wires for interconnect application, which is of great importance to Moore's law and semiconductor technology. However, the electronic transport physics of metallic SWNT bundles is more complex than that of a single tube. It results from the multitudes of numerous possible rotations of tubes with respect to each other, as well as the concomitant symmetry-breaking effects in presence of delicate coupling between the tubes. On the theoretical side, quantum transport properties of nanotube bundles are difficult to investigate from first principles because of the large number of atoms involved in a bundle, and it is the purpose of this work to fill this gap. In particular, we focus on metallic bundles made of armchair SWNTs and calculate their conductance spectra using a state-of-the-art $a b$ initio technique. We find the transport properties of bundles made of $(n, n)$ armchair SWNTs to be well classified into several categories depending on the chirality index $n$ of the tubes in the bundle. Each category is characterized by whether no gap, a partial gap, or a complete gap exists at the Fermi level of the conductance spectra. These gap structures are the di- rect consequences of geometric symmetry of the SWNTs inside the bundle, and they give substantial influence to the conductance of the bundle.

A bundle has more electronic conduction channels than a single nanotube. Without considering intertube coupling, the conductance of the bundle should simply scale with the number of tubes in the bundle. When there is tube-tube interaction, the coupling can induce symmetry breaking that mixes the $\pi$ and $\pi^{*}$ bands of the metallic armchair $(n, n)$ tubes in the bundle, leading to possible opening of band gaps near the Fermi level. Therefore the total conductance of a bundle may not be the simple summation of the conductance of all the participating tubes. Instead one may expect a dependence on how the tubes are orientated with respect to each other. The orientation becomes important because how adjacent tubes interact with each other dominates the electronic transport. Our work will systematically examine the characteristics of electronic structure and total energy of the bundles, and we correlate the transport properties to these characteristics in a general manner. The results can be explained qualitatively by symmetry considerations. Our quantum transport and bandstructure calculations of SWNT armchair bundles are accomplished by a first-principles real-space electron-transport package MATDCAL. ${ }^{8,9}$ In this technique, density-functional theory (DFT) is carried out with the Keldysh nonequilibrium Green's function (NEGF) formalism. The details of the NEGF-DFT formalism can be found elsewhere. ${ }^{10,11}$ In this work, we adopt standard nonlocal norm-conserving pseudopotentials $^{12}$ for the atomic cores and $s, p$, and $d$ single-zeta real-space basis set within the local-density approximation.

We calculated bundles with a finite cross section but having infinite length along the tube axis. For simplicity, individual tubes are assumed to have no impurities or defects so that all effects can be attributed to the coupling between the tubes. Unless stated otherwise, a nanotube bundle in this work contains seven nanotubes, with one tube in the middle surrounded by six other tubes forming a hexagonal pattern. This configuration mimics the close-packed structure of re- 


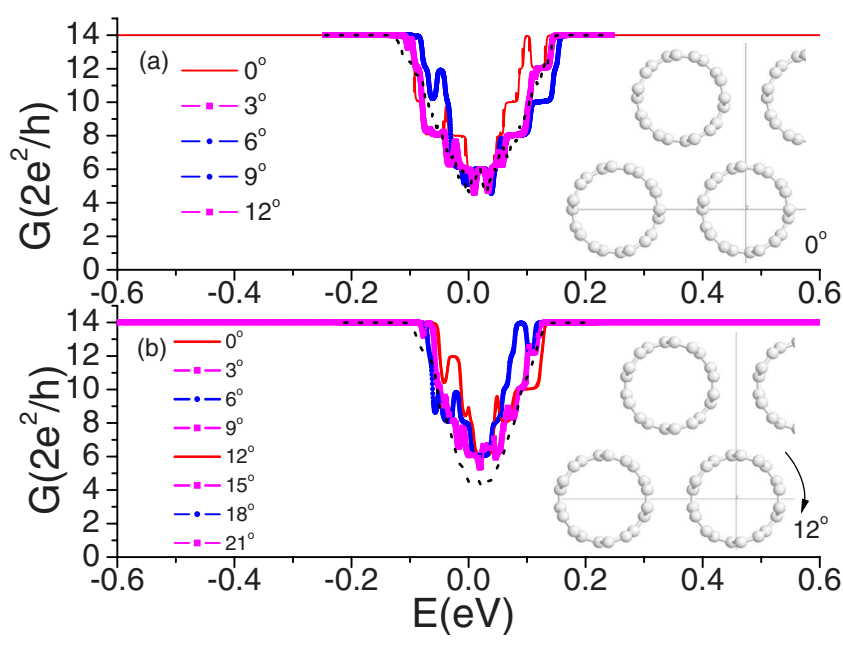

FIG. 1. (Color online) Electronic conductance of seven-tube bundles consisting of $(4,4)$ and $(5,5)$ SWNTs for different collective rotational angles (solid) and randomly orientated (dotted) bundles. Insets show the schematic end views of the SWNT bundles. Upper inset: the nanotubes are aligned along a horizontal axis in the crosssection plane; lower inset: all tubes are rotated by $12^{\circ}$ clockwise around tube axis collectively and categorized as an aligned bundle.

alistic nanotube bundles ${ }^{6}$ found in the experiments. The nearest wall-to-wall distance of two adjacent tubes is taken to be $3.33 \AA$ unless otherwise stated. Other kinds of bundles are also investigated (see below). We have studied bundles in which the tubes are aligned with various fixed rotational angles and bundles whose tubes are randomly orientated. In the aligned bundle, all the tubes are orientated with a certain angle with respect to a prespecified axis; in randomly orientated bundle, each tube has a different orientation angle drawn from a random-number generator. ${ }^{13}$ Finally, we have also employed the plane-wave basis DFT code VASP (Ref. 14) to calculate band structure and total energy of a periodic array of bundles. Energy cutoff of $194.0 \mathrm{eV}$ (14.26 Ry), projector augmented wave (PAW) potential, and CeperleyAlder (CA) local exchange-correlation functional were applied. The Brillouin zone was sampled with $6 \times 6 \times 12 k$ points of a Monkhorst-Pack grid. All the atoms in the unitcell and cell parameters were fully relaxed. The band structures obtained by VASP on the bulk are in good agreement with that obtained by MATDCAL on bundles with finite cross section.

We first consider the electric conductance of the $(4,4)$ and $(5,5)$ tube bundles. For a pristine single $(n, n)$ armchair metallic nanotube, there are two Bloch states crossing the Fermi level $E_{F}$ of the tube. Therefore in the ballistic transport regime one obtains a conductance $G=2 G_{0}$ for a single pristine armchair tube where the conductance quanta $G_{0}=2 e^{2} / h$. Since there are seven tubes in a bundle, we expect a total equilibrium conductance of $14 G_{0}$. Figure 1 shows that away from the immediate neighborhood of $E_{F}$, the calculated conductance indeed has a value of $14 G_{0}$. But within a sizable 0.2 $\mathrm{eV}$ range around $E_{F}$, the conductance of the bundle is strongly suppressed by a factor that can be up to $2 / 3$. Such a large suppression of the bundle's conductance is caused by intertube coupling which induces pseudogaps in the elec-

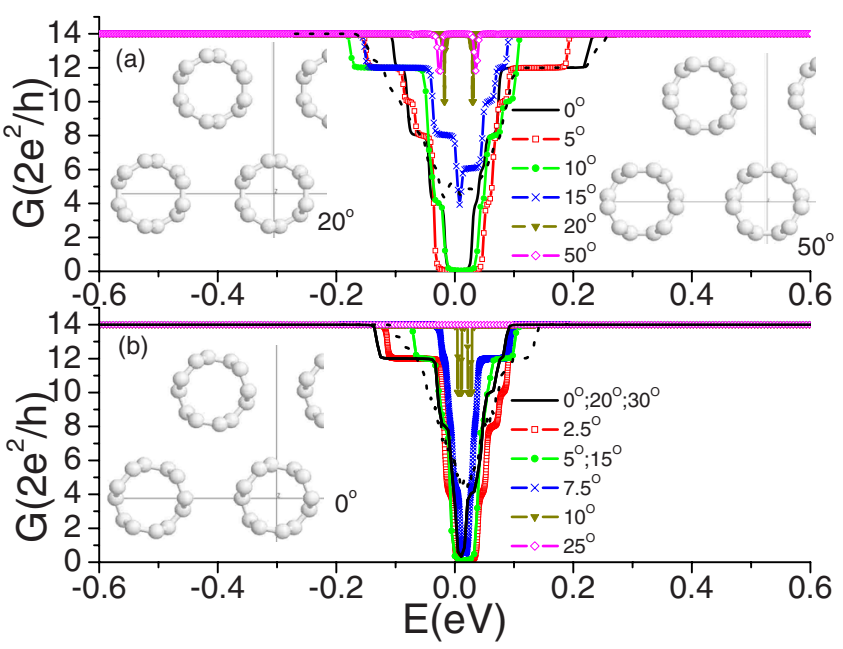

FIG. 2. (Color online) Conductance of bundles with $n=3 q$, $(3,3)$ and $(6,6) \mathrm{SWNT}$ bundles. (a) $20^{\circ}$ and $50^{\circ}$ for $(3,3)$ bundles and (b) $10^{\circ}$ and $50^{\circ}$ for $(6,6)$ bundles are gapless at the Fermi level. Other angles lead to either band gaps or pseudogaps at Fermi level. Dotted line: conductance spectra for averaged misaligned $(3,3)$ and $(6,6)$ bundles. Insets show the aligned tubes of different rotational angles for $(3,3)$ bundles.

tronic structure near the Fermi energy, ${ }^{3}$ leading to modified density of states (DOS) that reduces transmission coefficients of the conduction channels. Here we use the term pseudogap to indicate the situation shown in Fig. 1, where conductance is finite but has a value less than its maximum at $E_{F}$, e.g., $<14 G_{0}$ for seven-tube bundles. Figure 1 illustrates that the reduction in conductance $G$ displays universal behavior, independent of the details of the tube orientation inside the bundle. Furthermore, by averaging results from more than ten random configurations, the randomly orientated samples give almost the same conductance suppression spectra as that of the tubes aligned at specific angles (Fig. 1). We note that an individual $(n, n)$ tube with $n \neq 3 q$ ( $q$ =integer) has a different rotational symmetry around its axis compared with the $C_{3}$ rotational symmetry of the triangular lattice for the tubes inside a bundle. Therefore, our results on the $(4,4)$ and $(5,5)$ bundles suggest that $n \neq 3 q$ tube bundles have similar conductance suppression near the Fermi level regardless of how individual tubes are rotated with respect to each other, owing to the pseudogap opening induced by tube-tube coupling. This theoretical prediction and its inverse dependence on tube radius have been experimentally confirmed in a number of armchair nanotubes packed in bundles. ${ }^{4}$

When $n=3 q$, the individual $(n, n)$ tubes contain the $C_{3}$ rotational symmetry, which is compatible with the triangular lattice tube arrangement of the bundle. Such compatibility makes these bundles to behave very differently than the $n$ $\neq 3 q$ bundles. To be specific, we show the calculated conductance spectra of $(3,3)$ and $(6,6)$ SWNT bundles in Fig. 2. Depending on the rotational angle, the conductance spectra along the tube axis can have complete gaps, partial gaps, or no gap at all at Fermi level. If there is no gap, the bundle's conductance scales with the number of tubes inside the bundle, i.e., $G=14 G_{0}$ in bundle of seven tubes, reaching the maximal possible conductance. However, for $(3,3)$ and $(6,6)$ 
bundles having random tube orientations, the conductance spectra are rather similar to those of the $n \neq 3 q$ bundles shown in Fig. 1; the spectra of randomly orientated $(3,3)$ and $(6,6)$ bundles are plotted in Fig. 2 as dotted lines. This pseudogap is consistent with the previous experimental finding in $(9,9)$ bundle. ${ }^{4}$ However it does not exclude the possibility of observing our proposed zero and complete gaps in the future. The above anomalous results are generally observed in the bundles preserving threefold rotational symmetry, ${ }^{15,16}$ such as $(3,3),(6,6),(9,9)$ nanotubes and so on.

The complex and diversified transport characteristics for the $n=3 q$ bundles can be well understood from symmetry arguments. In particular, we find the opening or closing of band gaps to originate from atomistic level coupling by checking the detailed orientation configuration of the tubes in the bundle against the relevant electronic structures. There are two types of in-registry modes that allow maximum compatibility between the rotational symmetry of a tube around its axis and the global symmetry of the bundle's triangular lattice. If the symmetry of the single tube is compatible with the space group of the bundle lattice, the bundle can retain high symmetries for these specific orientations of the tubes, and thus the states crossing the Fermi level can transform according to different irreducible representations of the bundle space group. In the highest symmetry configurations, there are no avoided crossings for $\pi$ and $\pi^{*}$ bands near $E_{F}$, and the bundles attain maximal conductance. Other configurations with less compatibility have band-gap opening due to tube-tube interactions and if the gap is big enough (depending on the coupling strength), the number of available states at $E_{F}$ would be suppressed. We have confirmed this symmetry picture by additional studies of a hypothetical squarelattice bundle. The commensurability condition also requires compatible in-registry rotational angles of the individual tubes that lead to the possible conductance maximum for tubes with fourfold symmetries, such as $(4,4)$ tubes.

For any $(n, n)$ bundle with $n=3 q$ in a triangular lattice arrangement, we find the first two highest commensurate tubular rotational angles to be $60^{\circ} / n$ and $150^{\circ} / n$ (or equivalently $-30^{\circ} / n$ ). Therefore in Fig. 2 the $20^{\circ}$ and $50^{\circ}$ rotations for $(3,3)$ and the $10^{\circ}$ and $25^{\circ}$ rotations for $(6,6)$ bundles have no reduction in conductances at $E_{F}$ and their conductances reach the maximum $14 G_{0}$ at $E_{F}$. We identify the aligned $n$ $=3 q$ bundles (if $n$ is an even number) can attain high symmetries such as $\mathrm{P} 6 / \mathrm{MMM}(\mathrm{D} 6 \mathrm{H}-1)$ and $\mathrm{P} 6 / \mathrm{MCC}(\mathrm{D} 6 \mathrm{H}-2)$, while for aligned odd $n$ bundles, there can be $\mathrm{P} 63 /$ MMC(D6H-4) and P63/MCM(D6H-3) symmetries. For incommensurate out-of-registry angles, lower symmetries such as $\mathrm{P} 6 / \mathrm{M}(\mathrm{C} 6 \mathrm{H}-1)$ for even $n$ and $\mathrm{P} 63 / \mathrm{M}(\mathrm{C} 6 \mathrm{H}-2)$ for odd $n$ are found. Randomly orientated bundles for both even and odd $n$ generally have the lowest $\mathrm{P} 1(\mathrm{C} 1)$ symmetry only.

The conductance spectra are well correlated with the band structures. In a single isolated nanotube, the $\pi^{*}$ and $\pi$ bands cross each other at the Fermi point. In a nanotube bundle, each tube should contribute one pair (nondegenerate) $\pi^{*}$ and $\pi$ bands as shown in Fig. 3 for bundle with seven tubes. For a general given incommensurate collective rotational angle, e.g., $0^{\circ}(3,3)$ aligned bundle in Fig. 3(a), all the constituent bands cannot cross the Fermi level. These avoided crossings give rise to a complete band gap, and the bundle has zero
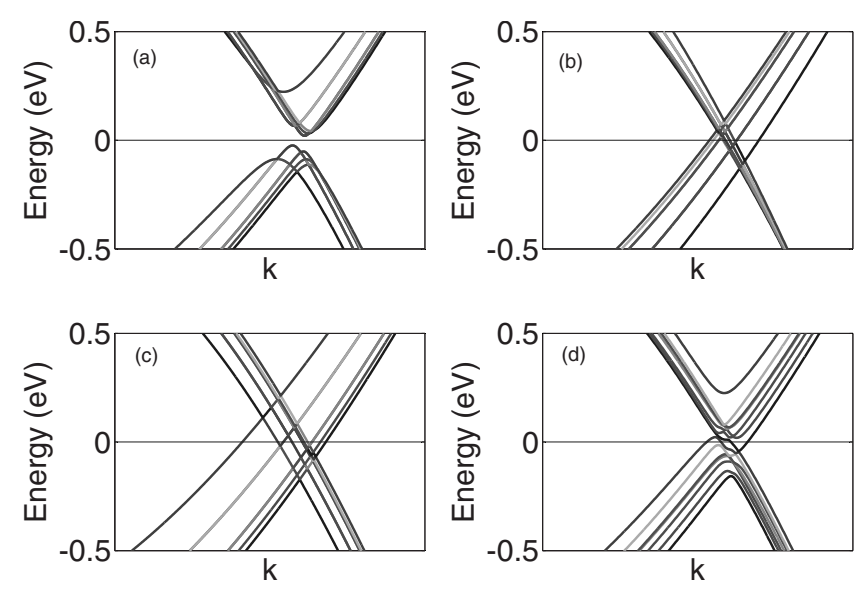

FIG. 3. Band structures for aligned $(3,3)$ bundle with rotational angle $0^{\circ}, 20^{\circ}, 50^{\circ}$, and randomly orientated [(a)-(d), respectively]. Complete and partial anticrossings of seven linear dispersion bands for the seven armchair SWNTs in the bundle are shown.

conductance. For the specific commensurate rotational angles such as $20^{\circ}$ and $50^{\circ}$ [Figs. 3(b) and 3(c)], the high symmetry allows the persistence of band crossings for all of the bands at $E_{F}$. The absence of band gap at $E_{F}$ allows for exactly $2 N$ $(N=7$ is the tube number) channels of conductance, leading to the maximum which is $N$ times that of a single tube. For randomly orientated bundles [Fig. 3(d)], there is a coexistence of band crossings and repulsions, showing a semimetallike density of states and one-third of maximal conductance at $E_{F}$. When $n \neq 3 q$, the bundles' band structures are not sensitive to the rotational angles for all bundles due to the general loss of commensurate symmetries in these tube bundles. This is the reason why similar DOS pseudogaps could be obtained for both aligned and misaligned $(10,10)$ SWNTs. ${ }^{3,17}$

Total energies calculated within DFT (Ref. 18) for $(3,3)$ and $(6,6)$ bundles exhibit strong dependence on the rotational angles of tubes, while those of $(4,4)$ and $(5,5)$ are nearly independent of such orientations, as shown in Fig. 4. For $(3,3)$ bundles, the two "gapless" angles have maximal $\left(50^{\circ}\right.$ or equivalently $\left.-10^{\circ}\right)$ and minimal $\left(20^{\circ}\right)$ energies, while for

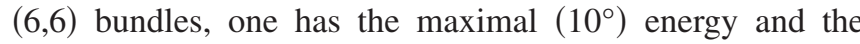
other $\left(25^{\circ}\right.$ or $\left.-5^{\circ}\right)$ has the second highest energy. We can trace this angle-dependent energetics to the delicate stacking of neighboring tube walls. ${ }^{15}$ The total energies depend on the closest wall areas of two neighboring tubes. We found that the high energy configurations generally correspond to an AA-like stacking of hexagons on tube walls facing to each other. The results shown in Fig. 4 also imply that if $n \neq 3 q$, the dependence of total energy on orientation is absent, in full agreement with the universal conductance suppression. While for the case of $n=3 q$, to reach the conductance maximum an alignment in some specific higher energy orientation can be necessary.

We also considered the effect of the bundle size and spacing between neighboring tubes (the latter is done in view of the fact that DFT may not be accurate in predicting the intertube distance of the bundle). The inset of Fig. 5 shows a nine-tube $(3,3)$ aligned $\left(0^{\circ}\right)$ bundle whose maximum conductance is $18 G_{0}$ and it has qualitatively the same conductance 


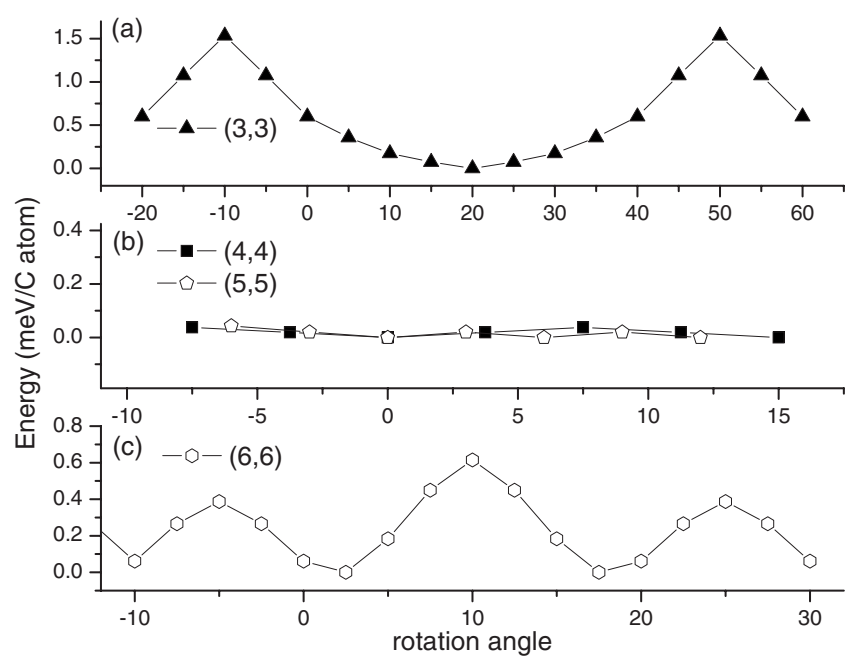

FIG. 4. Total energies per carbon atom for bundles are illustrated for $(3,3)-(6,6)$ tubes, relative to the lowest energy orientation (taken as zero energy reference).

spectra as the seven-tube bundle of Fig. 2(a). This suggests that the number of tubes in the bundle is not a determining factor compared with the symmetry. But the distance between tubes in a bundle strongly affects the coupling, which in turn controls the size of the gaps near $E_{F}$ and the conductance. As the tubes are separated farther away from each other, the conductance gap gradually closes up when the wall-to-wall distance is $\sim 5 \AA$ (not shown). This is expected because essentially the intertube coupling diminishes and results in the disappearance of the conductance gap. From Fig. 5 , it is interesting to observe that conductance of a finitesized bundle in the pseudogap region still undergoes quantized step changes, showing narrow conductance plateaus of integer multiples of $G_{0}=2 e^{2} / h$. In particular, our calculation has indicated that each of these conductance plateaus is characterized by a van Hove peak in DOS at energies corresponding to the conductance plateau edge. On the other hand, when infinite number of bundles forms a periodic crystal, DOS in the pseudogap region becomes smooth ${ }^{3}$ without the van Hove singularities of the one-dimensional small bundle considered here.

In conclusion, for useful applications of SWNT bundles in interconnect technology the conductance should be large and predictable (i.e., not dependent on details). We have identified the geometric conditions by which maximum conduc-

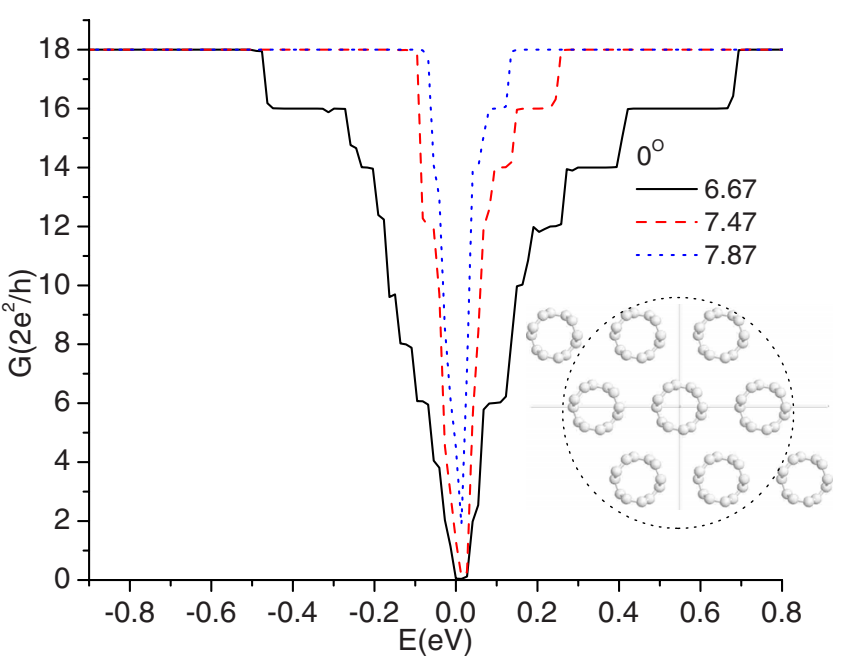

FIG. 5. (Color online) Conductance of relaxed bundles of nine $(3,3)$ tubes with varying tube lattice constants (center to center). The band gap is inversely proportional to the intertube spacing (rotational angle $0^{\circ}$ is shown).

tance can be obtained at the Fermi energy of the bundle. If the tubes are not of the $n=3 q$ type, the conductances are rather determined. From both electronic transport and energetic considerations, we obtain one-third conductance of $N$ single isolated tubes. In contrast when $n=3 q$ these conditions appear to be very delicate by depending on rotational angles of individual tubes and sometimes the most stable totalenergy configuration can correspond to nonoptimal conductance. Separating individual tubes laterally could eliminate this deterioration due to the bundling effects. On the other hand, one desires a small intertube distance for packing more tubes in a given area to enhance current density. These conflicting requirements are also crucial in the case if we pack semiconducting and metallic tubes into bundles containing a distribution of diameters or chiralities. ${ }^{19}$

W.R. is under financial support of HKUST through Grant No. RPC06/07.SC21. T.H.C. and T.C.L. acknowledge NCTS, financial support from NSC of Taiwan under Grant No. NSC-96-2112-M194-012-MY3, and computer time at the National Center for High Performance Computing. We also acknowledge financial support of HKSAR RGC under Grants No. CA04/05.SC02 (P.S.) and No. HKU 7048/06P (J.W.) and NSERC (H.G.). The computation resources were supported by the Shun Hing Education and Charity Fund. We thank Steven G. Louie for discussions.
${ }^{1}$ P. M. Ajayan and F. Banhart, Nature Mater. 3, 135 (2004).

${ }^{2}$ Y.-K. Kwon, S. Berber, and D. Tománek, Phys. Rev. Lett. 92, 015901 (2004).

${ }^{3}$ P. Delaney, H. J. Choi, J. Ihm, S. G. Louie, and M. L. Cohen, Nature (London) 391, 466 (1998).

${ }^{4}$ M. Ouyang, J.-L. Huang, C. L. Cheung, and C. M. Lieber, Science 292, 702 (2001).

${ }^{5}$ W. S. Su, T. C. Leung, B. Li, and C. T. Chan, Appl. Phys. Lett.
90, 163103 (2007).

${ }^{6}$ E. Einarsson, H. Shiozawa, C. Kramberger, M. H. Rummeli, A. Gruneis, T. Pichler, and S. Maruyama, J. Phys. Chem. C 111, 17861 (2007).

${ }^{7}$ Y. Awano, S. Sato, D. Kondo, M. Ohfuti, A. Kawabata, M. Nihei, and N. Yokoyama, Phys. Status Solidi A 203, 3611 (2006).

${ }^{8}$ D. Waldron, P. Haney, B. Larade, A. MacDonald, and H. Guo, Phys. Rev. Lett. 96, 166804 (2006). 
${ }^{9}$ W. Ren, J. R. Reimers, N. S. Hush, Y. Zhu, J. Wang, and H. Guo, J. Phys. Chem. C 111, 3700 (2007).

${ }^{10}$ J. Taylor, H. Guo, and J. Wang, Phys. Rev. B 63, 121104(R) (2001).

${ }^{11}$ J. Taylor, H. Guo, and J. Wang, Phys. Rev. B 63, 245407 (2001).

${ }^{12}$ D. R. Hamann, M. Schlüter, and C. Chiang, Phys. Rev. Lett. 43, 1494 (1979).

${ }^{13}$ We speculate that topological orientation transition could be possible in nanotube bundles, namely, a transition from random orientation to nonrandom orientation with a certain angle of each tube inside the bundle. Rotational ordering transition has been studied in two-dimensional (2D) C60 array by highresolution scanning tunneling microscopy at low temperature and the $\mathrm{C} 60$ solid does undergo a structural phase transition, i.e., from simple-cubic to face-centered-cubic phase transition above
249 K. [J. G. Hou, Jinlong Yang, Haiqian Wang, Qunxiang Li, Changgan Zeng, Lanfeng Yuan, Bing Wang, D. M. Chen, and Qingshi Zhu, Nature (London) 409, 304 (2001)].

${ }^{14}$ G. Kresse and J. Hafner, Phys. Rev. B 47, 558 (1993).

${ }^{15}$ J. C. Charlier, X. Gonze, and J. P. Michenaud, Europhys. Lett. 29, 43 (1995).

${ }^{16}$ S. Reich, C. Thomsen, and P. Ordejón, Phys. Rev. B 65, 155411 (2002).

${ }^{17}$ P. Delaney, H. J. Choi, J. Ihm, S. G. Louie, and M. L. Cohen, Phys. Rev. B 60, 7899 (1999).

${ }^{18}$ S. J. Sque, R. Jones, S. Oberg, and P. R. Briddon, Phys. Rev. B 75, 115328 (2007).

${ }^{19}$ A. A. Maarouf, C. L. Kane, and E. J. Mele, Phys. Rev. B 61, 11156 (2000). 